\title{
Lasing in nanoimprinted two-dimensional photonic crystal band-edge lasers
}

\author{
V. Reboud, ${ }^{1,2, a)}$ J. Romero-Vivas ${ }^{3}$ P. Lovera, ${ }^{4}$ N. Kehagias, ${ }^{1}$ T. Kehoe, ${ }^{1}$ G. Redmond,${ }^{5}$ \\ and C. M. Sotomayor Torres $\mathrm{s}^{1,6,7}$ \\ ${ }^{1}$ Catalan Institute of Nanotechnology, Campus UAB, Edifici CM3, ES 08193-Barcelona, Spain \\ ${ }^{2}$ CEA-LETI-Minatec Grenoble, 17 rue des Martyrs, 38054 Grenoble, France \\ ${ }^{3}$ Surface Physics Division, Faculty of Physics, Adam Mickiewicz University, ul. Umultowska 85, \\ Poznan 61-614, Poland \\ ${ }^{4}$ Tyndall National Institute, University College Cork, Lee Maltings, Cork, Ireland \\ ${ }^{5}$ School of Physics and School of Chemistry and Chemical Biology, University College Dublin, Belfield, \\ Dublin 4, Ireland \\ ${ }^{6}$ Catalan Institute for Research and Advanced Studies ICREA, 08010 Barcelona, Spain \\ ${ }^{7}$ Department of Physics, Universitat Autonoma de Barcelona, 08193 Bellaterra (Barcelona), Spain
}

(Received 3 August 2012; accepted 19 October 2012; published online 19 February 2013)

\begin{abstract}
We demonstrate optically pumped polymer band-edge lasers based on a two-dimensional photonic crystal slab fabricated by nanoimprint lithography (NIL). Lasing was obtained at the photonic band-edge, where the light exhibits a low group velocity at the $\Gamma$ point of the triangular lattice photonic crystal band structure. The active medium was composed of a dye chromophore-loaded polymer matrix directly patterned in a single step by nanoimprint lithography. Plane-wave and finite difference time domain algorithms were used to predict experimental lasing frequencies and the lasing thresholds obtained at different $\Gamma$ points. A low laser threshold of $3 \mu \mathrm{J} / \mathrm{mm}^{2}$ was achieved in a defect-free photonic crystal thus showing the suitability of nanoimprint lithography to produce cost-efficient optically pumped lasers. (C) 2013 American Institute of Physics.

[http://dx.doi.org/10.1063/1.4790646]
\end{abstract}

In recent years, polymer dye lasers have attracted considerable attention mainly due to the ease with which dyes may be incorporated in polymer, their capability to present a large tuneable emission by simply using different dyes, and the possibility to pattern polymers with cost-efficient fabrication processes over large areas for photonic and bio-sensing applications. So far, optically pumped organic lasers have been demonstrated over a large range of the visible spectra. ${ }^{1-6}$ These fabrication properties and optical characteristics when combined with the control of light by photonic crystal structures have led to study polymer photonic crystals $(\mathrm{PhC})$ band-edge lasers. They possess an intrinsic feedback mechanism based on Bloch waves near the PhC band edge leading to Bragg reflections induced by periodic structure, which reduce the group velocity of photons to zero at certain points of the band structure. Efficient distributed feedback is obtained by introducing a periodic modulation of the refractive index to the active material. Until now, efforts have been focused on demonstrating new functionalities of twodimensional photonic crystals, such as couplers, ${ }^{7}$ filters, ${ }^{8}$ waveguides, ${ }^{9}$ and lasers, ${ }^{10}$ using mature fabrication tools developed for silicon and semiconductor technology. Nanoimprint lithography (NIL) has been proposed as a candidate of high-resolution lithography for high volume and costeffective production over large areas with sub-10 $\mathrm{nm}$ resolution. ${ }^{11}$ NIL is a technique characterised by the unique ability to pattern active or functionalised polymer media in a single step. ${ }^{12,13}$ For instance, an important number of photonic components fabricated by NIL have recently been demonstrated, such as a wavelength filter based on a Bragg grating on a waveguide, ${ }^{14}$ a micro-ring resonator, ${ }^{15} 1 \mathrm{D}$ and $2 \mathrm{D}$

a)vincent.reboud@cea.fr. band-edge lasers, ${ }^{16-18}$ and photonic crystals for light extraction applications. ${ }^{19,20}$ In this work, we have achieved twodimensional polymer photonic crystal band-edge lasers fabricated by nanoimprint lithography in a dye-loaded printable polymer in a single fabrication step. The devices exhibit lasing oscillations at the different $\Gamma$-point band edge frequencies. Lasing frequencies predicted by plane wave expansion are in good agreement with the measured ones. The difference in lasing thresholds at the $\Gamma$ point band edge frequencies are explained by calculating the mode distribution overlap within the gain medium.

The polymer was loaded with dye by the simple process of mixing Rhodamine 6G (from Sigma Aldrich) with the printable polymer mr-NIL 6000 (from micro resist technol$o g y$ ) in two concentrations of $2.5 \times 10^{-3} \mathrm{~mol} / \mathrm{l}$ and $5 \times 10^{-3}$ mol/l. mr-NIL 6000 was chosen due to its relatively low glass transition temperature, around $45^{\circ} \mathrm{C}$, which enables low temperature printing and minimises the risk of degradation of the organic emitters during thermal NIL. ${ }^{21}$ The dyeloaded polymer was spin-coated on a glass substrate with a thickness of $400 \mathrm{~nm}$. The refractive index of the polymer was measured at a wavelength of $550 \mathrm{~nm}$ by ellipsometry to be 1.614. Silicon stamps were fabricated by electron beam lithography using a Jeol 6000 instrument. A dose of $130 \mu \mathrm{C} /$ $\mathrm{cm}^{2}$ and a beam current of $100 \mathrm{pA}$ were used with a $150 \mathrm{~nm}$ thick layer of ZEP 520 resist (Zeon Corporation) pre-baked at $120^{\circ} \mathrm{C}$ and developed during $30 \mathrm{~s}$ in a solution of $Z E D$ N50 (Zeon Corporation). The silicon stamp was etched to a depth of $350 \mathrm{~nm}$ using an inductively coupled plasma (ICP) reactive ion etching system (Surface Technology System) with a mixture of $\mathrm{SF}_{6}$ and $\mathrm{C}_{4} \mathrm{~F}_{8}$ gases. The stamp contained two triangular arrays of circular pillars with lattice constant of 500 and $580 \mathrm{~nm}$. It was treated with an anti-adhesive 


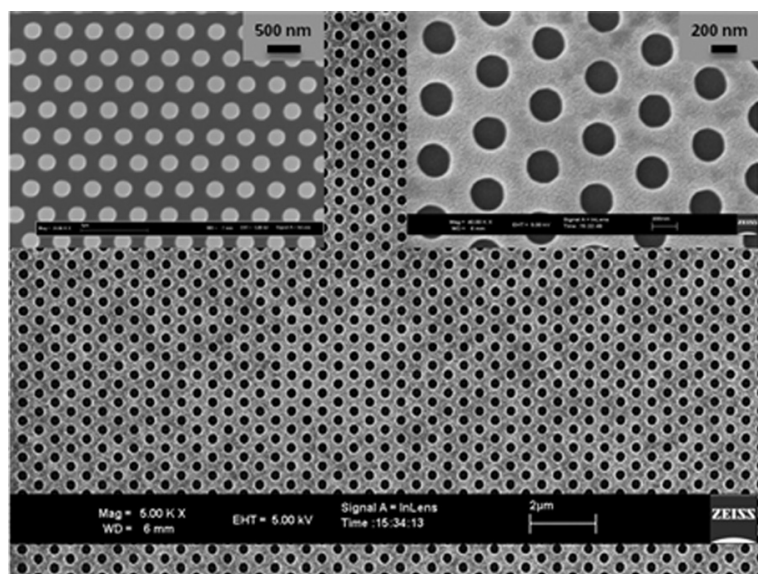

FIG. 1. SEM micrograph of nanoimprinted triangular lattice photonic crystals in dye-doped mr-NIL 6000 printable polymer. Inset: (left) SEM topview of one silicon stamp and (right) zoom of a dye-doped nanoimprinted photonic crystals.

monolayer of tridecafluor-1, 1, 2, 2-tetrahydrooctyl trichlorosilane deposited in the vapour phase, resulting in a low surface energy, ${ }^{22}$ to facilitate the release of the stamp from the polymer. Using a 2.5 in. Obducat nanoimprinting machine, the stamp was imprinted into the spin-coated polymer at a pressure of 60 bars for $5 \mathrm{~min}$ under a temperature of $90^{\circ} \mathrm{C}$. The pressure was sustained during cooling until the temperature reached $40^{\circ} \mathrm{C}$.

Photoluminescence (PL) was measured normal to the sample surface through a $10 \times$ microscope objective under laser excitation of $514.5 \mathrm{~nm}$ with a power of $200 \mu \mathrm{W}$. The dye-loaded polymer was imprinted with a non-structured stamp to test for changes to its optical properties due to the imprinting process. A 5\% decrease in the PL intensity of the dye loaded polymer was measured, for both concentrations, compared to freshly spin-coated films. SEM images of the 2D nanoimprinted PhCs in the dye chromophore-loaded polymer matrix are shown in Figure 1.
TABLE I. Comparison of the measured laser thresholds for the $\mathrm{PhC}$ bandedge lasers with the lattice constants $\mathrm{a}=500 \mathrm{~nm}\left(\Gamma_{1}\right)$ and $\mathrm{a}=580 \mathrm{~nm}\left(\Gamma_{3}\right)$ for the two dye concentrations.

\begin{tabular}{llc}
\hline \hline Dye concentration & \multicolumn{1}{c}{$\Gamma_{1}$} & $\Gamma_{3}$ \\
\hline $5 \times 10^{-3} \mathrm{~mol} / \mathrm{l}$ & $3 \mu \mathrm{J} / \mathrm{mm}^{2}$ & $3.9 \mu \mathrm{J} / \mathrm{mm}^{2}$ \\
$2.5 \times 10^{-3} \mathrm{~mol} / 1$ & $3.6 \mu \mathrm{J} / \mathrm{mm}^{2}$ & $4.7 \mu \mathrm{J} / \mathrm{mm}^{2}$ \\
\hline \hline
\end{tabular}

The samples were keep in a vacuum cell to reduce photobleaching of the dye and were optically pumped with a $1 \mathrm{~ns}$ pulse length $10 \mathrm{~Hz}$ frequency-doubled Q-switched $\mathrm{Nd}$ :VO4 laser light at $532 \mathrm{~nm}$ focused to a $40 \mu \mathrm{m}$ diameter spot on the sample surface. Figure 2 shows the measured emission spectra of the 2D PhCs with 500 and $580 \mathrm{~nm}$ lattice constants at different pump power, i.e., three times above the measured laser threshold for the $5 \times 10^{-3} \mathrm{~mol} / \mathrm{l}$ concentration nanoimprinted dye loaded polymer (Table I). The spectral laser linewidth at the full width at half maximum (FWHM) was measured to $1.1 \mathrm{~nm}$ above the threshold (Figures 2(a)-2(c)). This value is limited by the resolution of the spectrometer. The FWHM of the emission spectrum below the laser threshold is equal to $45 \mathrm{~nm}$ (Figure 2(d)). Figure 2(c) shows the emitted spectra of the $2 \mathrm{D} \mathrm{PhC}$ below the threshold and under a pulsed excitation of $17 \mu \mathrm{J} / \mathrm{mm}^{2}$. For a dye concentration of $5 \times 10^{-3} \mathrm{~mol} / \mathrm{l}$, the emission intensities were plotted as function of the incident excitation fluence for the laser with $500 \mathrm{~nm}$ lattice constant (inset of Figure 2(a)) and for the laser with $580 \mathrm{~nm}$ lattice constant (inset of Figure 2(a)). The light input-output relation exhibits a sharp turn at the laser threshold. Similar spectra were obtained for the polymer doped with a dye concentration of $2.5 \times 10^{-3} \mathrm{~mol} / \mathrm{l}$ showing the same lasing frequencies. At the photonic bandedge, the photon-matter interaction increases due to the significant reduction of the group velocity. To indentify bandedge frequencies, a plane wave algorithm from Optiwave Corporation was used to calculate the $2 \mathrm{D}$ dispersion relation of the $2 \mathrm{D} \mathrm{PhC}$ with a triangular lattice, for the transverse
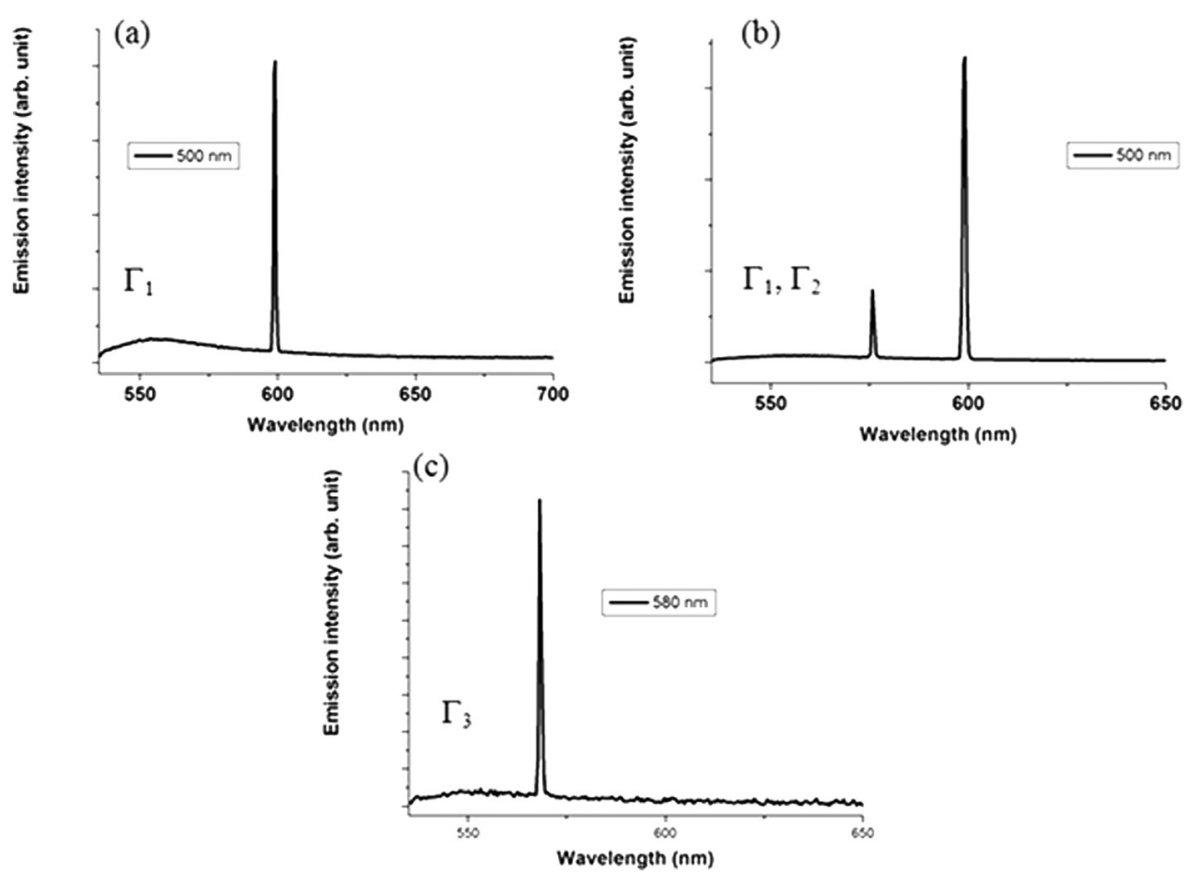

FIG. 2. Photoluminescence of $2 \mathrm{D} \mathrm{PhC}$ with a $500 \mathrm{~nm}$ lattice constant under a pulsed excitation of (a) $3.8 \mu \mathrm{J} / \mathrm{mm}^{2}$ (lasing at the $\Gamma_{1}$ point), and (b) solid line: $17 \mu \mathrm{J} / \mathrm{mm}^{2}$ (lasing at the $\Gamma_{1}$ and $\Gamma_{2}$ points), dot line: emission spectrum of Rhodamine $6 \mathrm{G}$ in mr-NIL 6000 below the laser threshold, (c) Photoluminescence of 2D PhC with a $580 \mathrm{~nm}$ lattice constant under a pulsed excitation of $16.5 \mu \mathrm{J} / \mathrm{mm}^{2}$ (lasing at the $\Gamma_{3}$ point). Dye concentration of $5 \times 10^{-3} \mathrm{~mol} / \mathrm{l}^{-1}$, d/ Emission spectrum of Rhodamine $6 \mathrm{G}$ in mrNIL 6000 below the laser threshold. Insets of (a) and (c): Peak emission intensity versus the absorbed excitation fluence associated to each $\mathrm{PhC}$ for a dye concentration of $5 \times 10^{-3} \mathrm{~mol} / \mathrm{l}$. 
electric (TE) modes. Transverse magnetic (TM) modes are expected to have higher losses because TM modes have a lower effective refractive index and, therefore, a lower confinement than TE modes.

The three first band edges can be identified at the $\Gamma$ point with reduced frequencies of $0.836\left(\Gamma_{1}\right), 0.868\left(\Gamma_{2}\right)$, and $1.021\left(\Gamma_{3}\right)$ (Figure 3(a)). The measured reduced frequencies, which are the ratio of the lasing frequencies to the lattice constant of the $\mathrm{PhCs}$, were calculated from Figure 2 to be $0.83,0.87$, and 1.03, respectively. Good agreement is obtained between simulation and experiments for the determination of lasing frequencies at the $\Gamma$ points.

Measured lasing thresholds for the two lattice constants at the two dye concentrations are shown in Table I. To understand the threshold difference at $\Gamma_{1}$ and at $\Gamma_{3}$ points, the magnetic field distribution normal to the array, $\mathrm{H}_{\mathrm{z}}$, for the band-edge mode at the two band edges was calculated by the finite-difference time domain (FDTD) method using perfectly matched layer boundaries in all directions.

An amplitude profile of the two band-edge modes is formed over the PhC area as shown on Figure 3(b). The black circles in both pairs of the Figure 3(b) denote the air-hole boundary and the area outside the circles corresponds to the dye-doped polymer. Part of the mode energy at the $\Gamma_{3}$ point overlaps with the holes in the array where there is no gain medium and consequently this energy is lost. The overlap of the modes with the gain medium is calculated for the two modes with the band edges at the $\Gamma_{1}$ and $\Gamma_{3}$ points. A $31.6 \%$ difference in the overlap is obtained for the two modes explaining the higher threshold for the mode at the $\Gamma_{3}$ point. This value is slightly over estimated in comparison to the calculated difference of $23 \%$ between the lasing threshold at the $\Gamma_{1}$ and the $\Gamma_{3}$ points for the two dye concentrations (Table I) since the residual layer is not taking in account in the simulation. a/

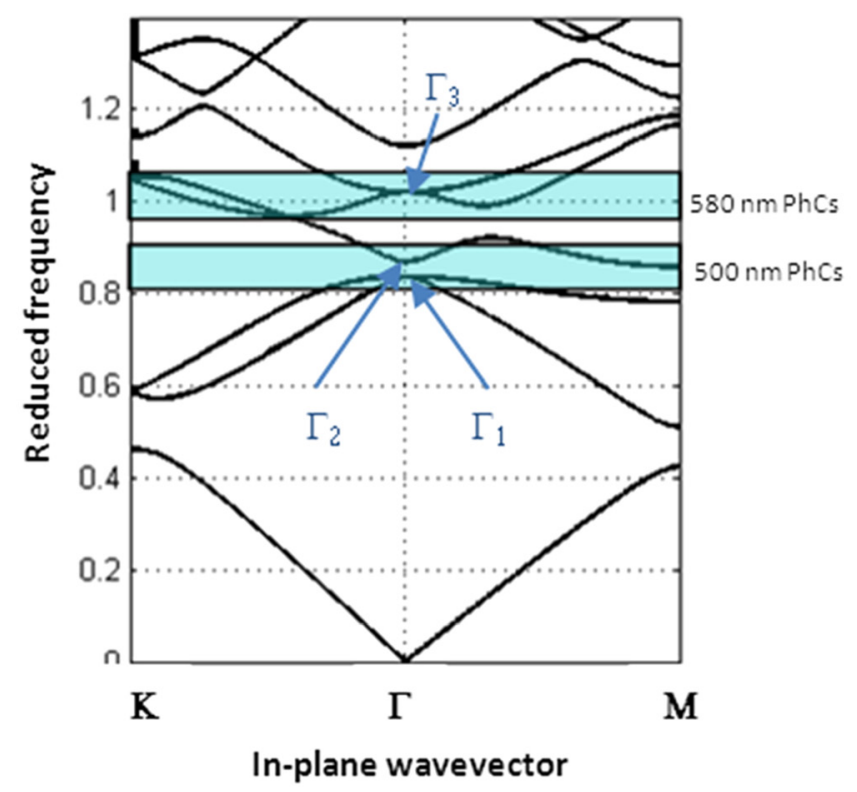

b/
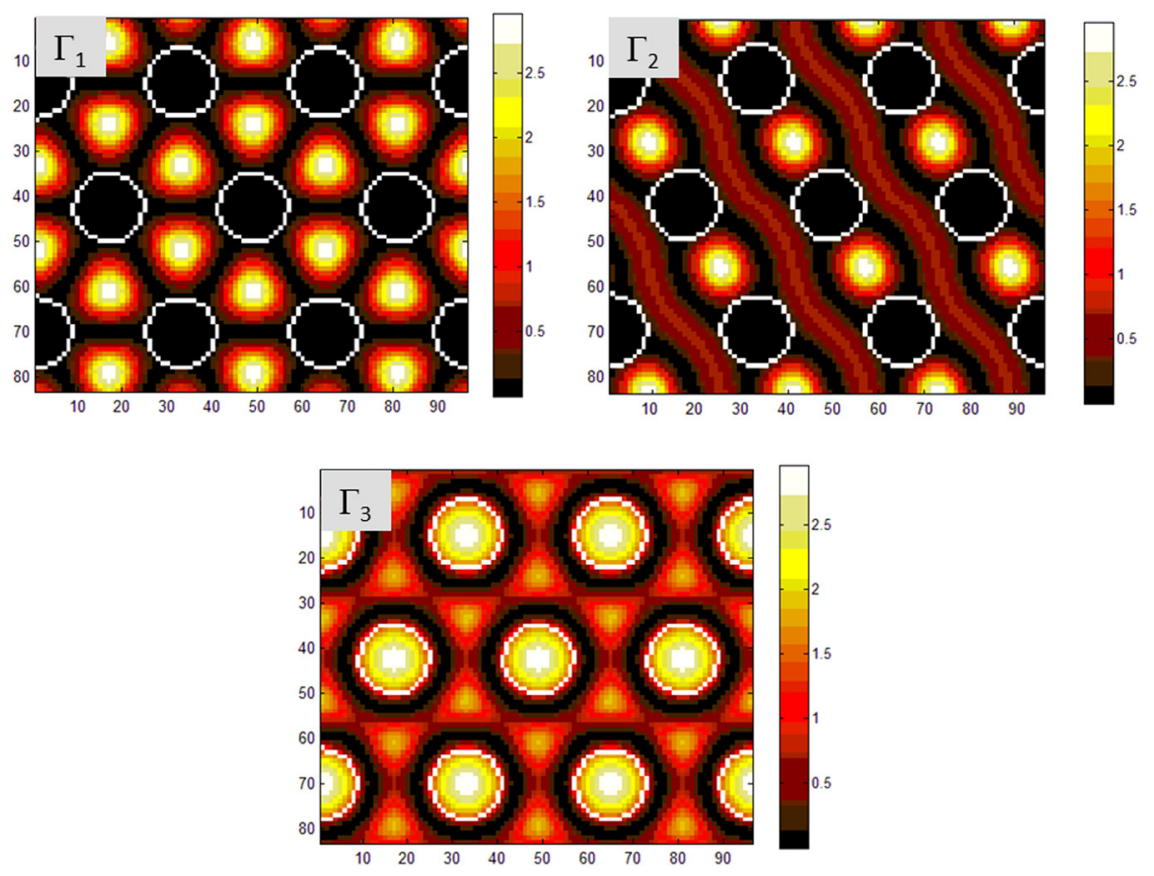

FIG. 3. (a) Photonic band structure of a triangular lattice 2D air hole $\mathrm{PhC}$ calculated with a Plane wave-basis frequency-domain method for TE polarization where $a$ is the lattice constant and $\lambda$ is the wavelength. Hole radius is $R=0.24 a$. The bands represent the gain bandwidth of Rhodamine $6 \mathrm{G}$ for $\mathrm{PhC}$ lattice constants $a=500 \mathrm{~nm}$ and $a=580 \mathrm{~nm}$, respectively. (b) Calculated $\mathrm{H}_{\mathrm{z}}$ magnetic-field density at the $\Gamma_{1}, \Gamma_{2}$, and $\Gamma_{3}$ points. 


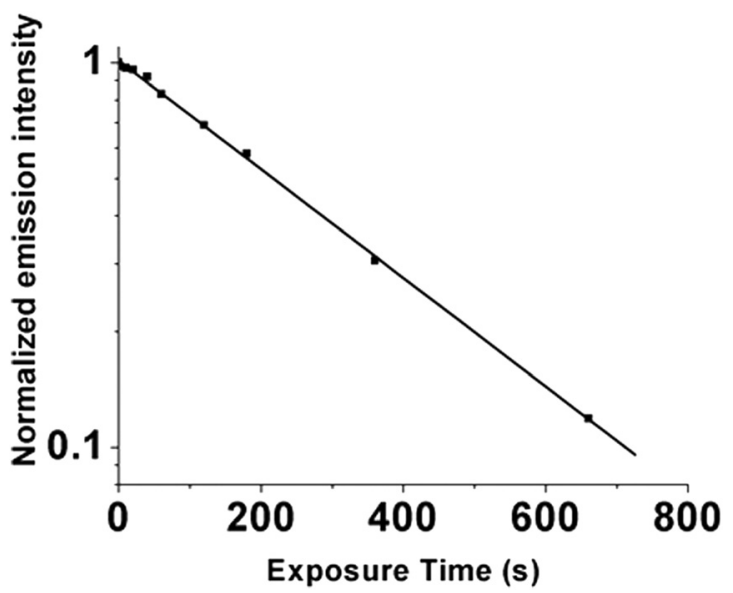

FIG. 4. Laser lifetime increasing the exposure time under pumping power of 2.8 the threshold level $\left(8.5 \mu \mathrm{J} / \mathrm{mm}^{2}\right.$ and pulse frequency: $\left.10 \mathrm{~Hz}\right)$.

Finally, the lifetime of the band-edge laser was investigated by recording repeatedly the lasing intensity at the $\Gamma_{1}$ point of the $500 \mathrm{~nm}$ pitch $\mathrm{PhCs}$ in a vacuum cell. Figure 4 shows the lifetime of the laser as a function of time, at a rate of 10 pulses per second at $8.5 \mu \mathrm{J} / \mathrm{mm}^{2}$, which corresponds to 2.8 times the laser threshold. The short lifetime of the $2 \mathrm{D}$ $\mathrm{PhC}$ band-edge lasers is due to photobleaching of the Rhodamine 6G. To increase the lifetime of the lasers, the dye molecules need to be replaced by more robust luminescence sources, for example, by semiconductor nanocrystals with optical gain, ${ }^{23}$ embedded in the printable polymer.

In conclusion, we have demonstrated optically pumped lasing in two-dimensional polymer photonic crystal bandedge lasers operating in the visible range fabricated in a single step by nanoimprint lithography. Lasing was identified at three photonic band-edges $\left(\Gamma_{1}, \Gamma_{2}\right.$, and $\left.\Gamma_{3}\right)$. The measured lasing frequencies are in good agreement with simulations. The difference in lasing threshold between modes at the $\Gamma_{1}$ and $\Gamma_{3}$ band-edges can be predicted by calculating the lasing mode overlap with the patterned area. We showed that nanoimprint lithography is suitable for the fabrication of costefficient, compact, and high quality surface emitting photonic crystal band-edge lasers. In the future, our work will focus on the study of light conversion performance of nanoimprinted band edge lasers and the characteristics of the emitted laser beam.
We gratefully acknowledge Mads Brøkner Christiansen and Anders Kristensen for their help with dye-doping printable polymer. The support of the EC-funded project $\mathrm{NaPa}$ NIL is gratefully acknowledged. The content of this work is the sole responsibility of the authors.

${ }^{1}$ T. Rabe, M. Hoping, D. Schneider, E. Becker, H. H. Johannes, W. Kowalsky, T. Weimann, J. Wang, P. Hinze, B. S. Nehls, U. Scherf, T. Farrell, and T. Riedl, Adv. Funct. Mater. 15, 1188 (2005).

${ }^{2}$ P. Gorrn, T. Rabe, T. Riedl, W. Kowalsky, F. Galbrecht, and U. Scherf, Appl. Phys. Lett. 89, 161113 (2006).

${ }^{3}$ H. Nakanotani, S. Akiyama, D. Ohnishi, M. Moriwake, M. Yahiro, T. Yoshihara, S. Tobita, and C. Adachi, Adv. Funct. Mater. 17, 2328 (2007).

${ }^{4}$ J. R. Lawrence, E. B. Namdas, G. J. Richards, P. L. Burn, and I. D. W. Samuel, Adv. Mater. 19, 3000 (2007).

${ }^{5}$ I. D. W. Samuel and G. A. Turnbull, Chem. Rev. 107, 1272 (2007).

${ }^{6}$ B. K. Yap, R. D. Xia, M. Campoy-Quiles, P. N. Stavrinou, and D. D. C. Bradley, Nature Mater. 7, 376 (2008).

${ }^{7}$ S. Boscolo, M. Midrio, and T. F. Krauss, Opt. Lett. 27, 1001 (2002).

${ }^{8}$ Z. X. Qiang, W. D. Zhou, and R. A. Soref, Opt. Express 15, 1823-1831 (2007).

${ }^{9}$ G. Chen and J. U. Kang, Opt. Lett. 30, 1656-1658 (2005).

${ }^{10}$ D. Englund, H. Altug, I. Fushman, and J. Vuckovic, Appl. Phys. Lett. 91, 071126 (2007).

${ }^{11}$ S. Y. Chou, P. R. Krauss, and P. J. Resnstrom, Appl. Phys. Lett. 67, 3114 (1995).

${ }^{12}$ A. Genua, J. A. Alduncín, J. A. Pomposo, H. Grande, N. Kehagias, V. Reboud, C. M. Sotomayor, I. Mondragon, and D. Mecerreyes, Nanotechnology 18, 215301 (2007).

${ }^{13}$ V. Reboud, A. Z. Khokhar, B. Sepúlveda, D. Dudek, T. Kehoe, J. Cuffe, N. Kehagias, M. Lira-Cantu, N. Gadegaard, V. Grasso, V. Lambertini and C. M. Sotomayor Torres, Nanoscale 4, 3495-3500 (2012).

${ }^{14}$ S.-W. Ahn, K.-D. Lee, D.-H. Kim, and S.-S. Lee, IEEE Photon. Technol. Lett. 17(10), 2122-2124 (2005).

${ }^{15}$ D.-H. Kim, J.-G. Im, S.-S. Lee, S.-W. Ahn, and K.-D. Lee, IEEE Photon. Technol. Lett. 17(11), 2352-2354 (2005).

${ }^{16}$ C. L. C. Smith, J. U. Lind, C. H. Nielsen, M. B. Christiansen, T. Buss, N. B. Larsen, and A. Kristensen, Opt. Lett. 36, 1392-1394 (2011).

${ }^{17}$ E. B. Namdas, M. Tong, P. Ledochowitsch, S. R. Mednick, J. D. Yuen, D. Moses, and A. J. Heeger, Adv. Mater. 21, 799-802 (2009).

${ }^{18}$ V. Reboud, P. Lovera, N. Kehagias, M. Zelsmann, C. Schuster, F. Reuther, G. Gruetzner, G. Redmond, and C. M. Sotomayor Torres, Appl. Phys. Lett. 91, 151101 (2007).

${ }^{19}$ V. Reboud, N. Kehagias, M. Striccoli, T. Placido, A. Panniello, M. L. Curri, M. Zelsmann, F. Reuther, G. Gruetzner, and C. M. Sotomayor Torres, J. Vac. Sci. Technol. B 25, 2642-2644 (2007).

${ }^{20}$ N. Kehagias, V. Reboud, G. Chansin, M. Zelsmann, C. Jeppesen, F. Reuther, C. Schuster, M. Kubenz, G. Gruetzner, and C. M. Sotomayor Torres, J. Vac. Sci. Technol. B 24, 3002-3005 (2006).

${ }^{21}$ F.-H. Ko, L.-Y. Weng, C.-J. Ko, and T.-C. Chu, Microelectron. Eng. 83, 864 (2006).

${ }^{22}$ M. Bender, M. Otto, B. Hadam, B. Spangenberg, and H. Kurz, Microelectron. Eng. 61-62, 407 (2002).

${ }^{23}$ V. I. Klimov, S. A. Ivanov, J. Nanda, M. Achermann, I. Bezel, J. A. McGuire, and A. Piryatinski, Nature 447, 441 (2007). 